\title{
Thermal Non-Equilibrium Forced Convective Flow in an Annulus Filled with a Porous Medium
}

\author{
C. Yang ${ }^{*}, 1,2$, F. Kuwahara ${ }^{1}$, W. Liu ${ }^{2}$ and A. Nakayama ${ }^{1,3}$ \\ ${ }^{I}$ Department of Mechanical Engineering, Shizuoka University, 3-5-1 Johoku, Naka-Ku, Hamamatsu, 432-8561 Japan \\ ${ }^{2}$ School of Energy and Power Engineering, Huazhong University of Science and Technology, Wuhan, Hubei 430074, PR \\ China \\ ${ }^{3}$ School of Civil Engineering and Architecture, Wuhan Polytechnic University, Wuhan, Hubei 430023, China
}

\begin{abstract}
In light of a local thermal non-equilibrium model, fully developed forced convection in an annulus filled with a porous medium is treated to elucidate its exact temperature solutions for both fluid and solid phases. In this study, the plug-flow approximation is assumed since Darcian velocity over the cross-section is uniform except in a small region very close to the walls. The inner wall is heated under the constant heat flux condition while the outer wall is subject to the adiabatic boundary condition. The exact solutions based on the present local thermal non-equilibrium model clearly show that the local thermal equilibrium assumption may fail for the case of metal foam and air combination. The approximate solution for the Nusselt number, which is in accord with the corresponding exact solution, has also been presented for possible engineering applications.
\end{abstract}

Keywords: Porous media, thermal non-equilibrium, aluminum, metal foam, annulus.

\section{INTRODUCTION}

Two kinds of models, namely, thermal equilibrium model and thermal non-equilibrium model, can be applied to investtigate thermal characteristics of conduction and convection within a porous medium. The only difference between these two models is whether the local thermal equilibrium is assumed or not. A substantial number of researchers [1-3] adopted the thermal equilibrium model to perform the theoretical and numerical investigations. Nevertheless, the local thermal equilibrium assumption between the solid and fluid phase is often inappropriate for a number of problems as pointed out by Carbonnel and Whitaker [4], Vafai and Sozen [5], Quintard and Whitaker [6, 7], Quintard [8], Amiri and Vafai [9], Jiang et al. [10], Jiang and Ren [11], Peterson and Chang [12], and Spiga and Morina [13], who recommended the use of local thermal non-equilibrium model.

Noticeable contributions have been made for the improvement of local thermal non-equilibrium model. Alazmi and Vafai [14] analyzed the effect of variant boundary conditions for the case of constant wall heat flux based on the local thermal non-equilibrium model. Meanwhile, pertinent parameters such as the porosity, Reynolds number, Darcy number, inertia parameter, particle diameter, and the ratio of solid to fluid conductivity are taken into account in their research. Finally, they proposed a useful and comprehensive set of correlations that relate these parameters with the total Nusselt number. Under the assumption of local

*Address correspondence to this author at the Department of Mechanical Engineering, Shizuoka University, 3-5-1 Johoku, Naka-Ku, Hamamatsu, 432-8561 Japan; Tel: +81-53-478-1049; Fax: +81-53-478-1049; E-mail: f5945037@ipc.shizuoka.ac.jp thermal non-equilibrium, Jiang and Ren [11] numerically performed the comprehensive calculations in which the effects of viscous dissipation, the boundary condition assumptions, thermal dispersion, particle diameters and the variable properties of oil on heat transfer characteristics are considered. A good agreement between the predicted convection heat transfer coefficients and the corresponding experimental results indicates that the utilization of local thermal non-equilibrium model is essential.

By using two energy equations introduced by Hsu [15] and Hsu et al. [16], Nakayama et al. [17] presented exact solutions for two fundamental steady heat transfer cases, namely, one-dimensional steady heat conduction in a porous slab with internal heat generation, and also thermally developing unidirectional flow through a semi-infinite porous medium. However, exact solutions for such local thermal non-equilibrium problems are very much limited, because of the analytical difficulty in solving the two energy equations simultaneously. These exact solutions are essentially needed for possible benchmark tests of numerical tools based on the thermal non-equilibrium assumption.

In this study, we shall consider a fundamental yet important case in the field of engineering applications, namely, the fully developed forced convection in an annulus filled with a porous medium. Exact solutions for the case of aluminum foam and air combination are sought for the annulus in which the inner wall surface is heated by constant heat flux whereas the outer wall surface is insulated. We shall consider the effects of tortuosity on the stagnant conductivity on the heat transfer characteristics, which were often neglected in previous investigations. It will be shown that the local thermal equilibrium assumption may fail for the constant heat flux case in which the fluid and solid 
phases within the gap of annular tube are never at thermal equilibrium.

\section{MATHEMATIC MODEL BASED ON LOCAL THERMAL NON-EQUILIBRIUM ASSUMPTION}

Upon integrating two energy equations for the two individual phases over a representative elementary volume $V$ following the volume averaging theory $[6,7,18,19]$, we obtain the macroscopic energy equations for the two individual phases as follows:

For the fluid phase:

$$
\begin{aligned}
& \varepsilon \rho_{f} c_{p_{f}} \frac{\partial\langle T\rangle^{f}}{\partial t}+\varepsilon \rho_{f} c_{p_{f}} \frac{\partial\left\langle u_{j}\right\rangle^{f}\langle T\rangle^{f}}{\partial x_{j}} \\
&=\frac{\partial}{\partial x_{j}}\left(\varepsilon k_{f} \frac{\partial\langle T\rangle^{f}}{\partial x_{j}}+\frac{k_{f}}{V} \int_{A_{\text {int }}} T n_{j} d A-\rho_{f} c_{p_{f}} \varepsilon\left\langle\tilde{u}_{j} \tilde{T}\right\rangle^{f}\right) \\
&+\frac{1}{V} \int_{A_{\text {int }}} k_{f} \frac{\partial T}{\partial x_{j}} n_{j} d A
\end{aligned}
$$

For the solid matrix phase:

$$
\begin{aligned}
\rho_{s} c_{s}(1-\varepsilon) & \frac{\partial\langle T\rangle^{s}}{\partial t} \\
=\frac{\partial}{\partial x_{j}}\left((1-\varepsilon) k_{s} \frac{\partial\langle T\rangle^{s}}{\partial x_{j}}\right. & \left.-\frac{k_{s}}{V} \int_{A_{\text {int }}} T n_{j} d A\right) \\
& -\frac{1}{V} \int_{A_{\text {int }}} k_{f} \frac{\partial T}{\partial x_{j}} n_{j} d A
\end{aligned}
$$

where the volume average of a certain variable $\varphi$ in the fluid phase is defined as,

$$
\langle\varphi\rangle^{f} \equiv \frac{1}{V_{f}} \int_{V_{f}} \varphi d V
$$

such that $\langle T\rangle^{f}$ is the intrinsic volume average of the fluid temperature, while $\langle T\rangle^{s}$ is the intrinsic volume average of the solid matrix temperature, where $V_{f}$ is the volume space which the fluid phase occupies. The porosity $\varepsilon \equiv V_{f} / V$ is the volume fraction of the fluid space. The variable $\varphi$ is decomposed into its intrinsic average and the spatial deviation from it:

$$
\varphi=\langle\varphi\rangle^{f}+\tilde{\varphi}
$$

Moreover, $A_{\text {int }}$ is the local interfacial area between the fluid and solid matrix, while $n_{i}$ is the unit vector pointing outward from the fluid side to solid side. The continuity of both temperature and heat flux is imposed on the interface. Obviously, the parenthetical terms on the right hand-side of Equation (1) denote the diffusive heat transfer, while the last term describes the interfacial heat transfer between the solid and fluid phases. In terms of the local thermal equilibrium assumption, the following one-equation models can be achieved by combining the previous Equations (1) and (2):

$$
\begin{aligned}
& \left(\varepsilon \rho_{f} c_{p_{f}}+(1-\varepsilon) \rho_{s} c_{s}\right) \frac{\partial\langle T\rangle}{\partial t}+\rho_{f} c_{p_{f}} \frac{\partial\left\langle u_{j}\right\rangle\langle T\rangle}{\partial x_{j}} \\
& =\frac{\partial}{\partial x_{j}}\left(\begin{array}{c}
\left(\varepsilon k_{f}+(1-\varepsilon) k_{s}\right) \frac{\partial\langle T\rangle}{\partial x_{j}} \\
+\frac{k_{f}-k_{s}}{V} \int_{A_{\text {int }}} T n_{j} d A-\varepsilon \rho_{f} c_{p_{f}}\left\langle\tilde{u}_{j} \tilde{T}\right\rangle^{f}
\end{array}\right)
\end{aligned}
$$

Where,

$$
\langle\varphi\rangle \equiv \frac{1}{V} \int_{V} \varphi d V
$$

is the Darcian average of the variable $\varphi$ such that $\left\langle u_{j}\right\rangle=\varepsilon\left\langle u_{j}\right\rangle^{f}$ is the Darcian velocity vector. From the foregoing equation (3), the macroscopic heat flux vector $q_{i}=\left(q_{x}, q_{y}, q_{z}\right)$ and its corresponding stagnant thermal conductivity $k_{\text {stag }}$ may be defined as follows:

$$
\begin{aligned}
q_{i}=-k_{\text {stag }} & \frac{\partial\langle T\rangle}{\partial x_{i}}+\varepsilon \rho_{f} c_{p_{f}}\left\langle\tilde{u}_{i} \tilde{T}\right\rangle^{f} \\
= & -\left(\varepsilon k_{f}+(1-\varepsilon) k_{s}\right) \frac{\partial\langle T\rangle}{\partial x_{i}} \\
& -\left(k_{f}-k_{s}\right) \frac{1}{V} \int_{A_{\text {int }}} T n_{i} d A+\varepsilon \rho_{f} c_{p_{f}}\left\langle\tilde{u}_{i} \tilde{T}\right\rangle^{f}
\end{aligned}
$$

Note that the first term in the rightmost expression corresponds to the upper bound of the effective stagnant thermal conductivity based on the parallel model, namely, $\left(\varepsilon k_{f}+(1-\varepsilon) k_{s}\right)$. Thus, it is the tortuosity term (i.e. the second term) that adjusts the level of the effective stagnant thermal conductivity from its upper bound to a correct one.

Meanwhile, for obtaining more concise and neat forms of previous two energy equations, the following two-energy equation model was presented by Yang and Nakayama [20] along with the effective porosity:

For the fluid phase:

$$
\begin{gathered}
\rho_{f} c_{p_{f}} \varepsilon \frac{\partial\langle T\rangle^{f}}{\partial t}+\rho_{f} c_{p_{f}} \frac{\partial\left\langle u_{j}\right\rangle\langle T\rangle^{f}}{\partial x_{j}} \\
=\frac{\partial}{\partial x_{j}}\left(\left(\varepsilon^{*} k_{f} \frac{\partial\langle T\rangle^{f}}{\partial x_{j}}+\varepsilon k_{d i s_{j k}} \frac{\partial\langle T\rangle^{f}}{\partial x_{k}}\right)\right) \\
-h_{v}\left(\langle T\rangle^{f}-\langle T\rangle^{s}\right)
\end{gathered}
$$

For the solid matrix phase:

$$
\begin{aligned}
& \rho_{s} c_{s}(1-\varepsilon) \frac{\partial\langle T\rangle^{s}}{\partial t} \\
& =\frac{\partial}{\partial x_{j}}\left(\left(1-\varepsilon^{*}\right) k_{s} \frac{\partial\langle T\rangle^{s}}{\partial x_{j}}\right)-h_{v}\left(\langle T\rangle^{s}-\langle T\rangle^{f}\right)
\end{aligned}
$$


where the effective porosity $\varepsilon^{*}$ which accounts for the effect of tortuosity on the stagnant thermal conductivity is defined such that the stagnant thermal conductivity is given by,

$k_{\text {stag }}=\varepsilon^{*} k_{f}+\left(1-\varepsilon^{*}\right) k_{s}$

namely,

$\varepsilon^{*}=\frac{k_{s}-k_{\text {stag }}}{k_{s}-k_{f}}=\varepsilon+\frac{\varepsilon k_{f}+(1-\varepsilon) k_{s}-k_{\text {stag }}}{k_{s}-k_{f}}$

such that Equation (4) gives,

$\left(\varepsilon^{*}-\varepsilon\right) \frac{\partial\langle T\rangle}{\partial x_{i}}=\frac{1}{V} \int_{A_{\text {int }}} T n_{i} d A$

As the stagnant thermal conductivity $k_{\text {stag }}$ is given either empirically or theoretically, the effective porosity $\varepsilon^{*}$ can easily be evaluated from (7b). Furthermore, the thermal dispersion term is modeled according to the gradient diffusion hypothesis [21]:

$-\rho_{f} c_{p f}\left\langle\tilde{u} \tilde{T}_{j} \tilde{T}\right\rangle^{f}=k_{d i s_{k j}} \frac{\partial\langle T\rangle^{f}}{\partial x_{k}}$

while the interfacial heat transfer between the solid and fluid phases is modeled using Newton's cooling law:

$\frac{1}{V} \int_{A_{\text {int }}} k_{f} \frac{\partial T}{\partial x_{j}} n_{j} d A=h_{v}\left(\langle T\rangle^{s}-\langle T\rangle^{f}\right)$

where $h_{v}$ is the volumetric heat transfer coefficient.

\section{PHYSICAL MODEL}

We shall seek possible exact solutions for convective heat transfer in an annulus filled with metal foams, using the foregoing thermal non-equilibrium model. As indicated in Fig. (1), the air is flowing through an infinitely long annulus, the gap of which is fully filled with a metal foam. The inner wall surface is heated by constant heat flux whereas the outer wall surface is insulated. As pointed out by Dukhan et al. [22], the Darcian velocity shows its dependence on the transverse direction only in a small region very close to the wall. Therefore, we may neglect the boundary term (i.e. Brinkman term) and use the plug-flow approximation. Under this approximation, sufficiently away from the entrance, the energy equations (5) and (6) for the individual phases reduce to the following differential equations:

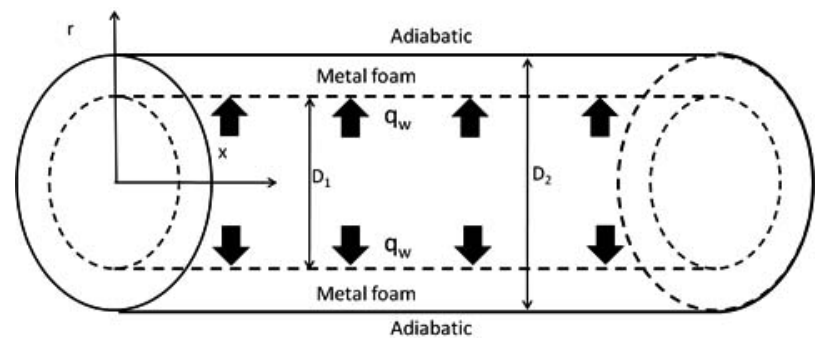

Fig. (1). Convective heat transfer in anannulus filled with a metal foam.
For the fluid phase:

$$
\begin{aligned}
\rho_{f} c_{p_{f}} u_{D} & \frac{\partial\langle T\rangle^{f}}{\partial x} \\
=\left(\varepsilon^{*} k_{f}+\varepsilon k_{d i s y}\right) \frac{1}{r} \frac{\partial}{\partial r} & \left(r \frac{\partial\langle T\rangle^{f}}{\partial r}\right) \\
& -h_{v}\left(\langle T\rangle^{f}-\langle T\rangle^{s}\right)
\end{aligned}
$$

For the solid phase:

$$
\left(1-\varepsilon^{*}\right) k_{s} \frac{1}{r} \frac{\partial}{\partial r}\left(r \frac{\partial\langle T\rangle^{s}}{\partial r}\right)-h_{v}\left(\langle T\rangle^{s}-\langle T\rangle^{f}\right)=0
$$

with the following boundary conditions:

$q_{w}=-\left[\begin{array}{c}\left.\left(\varepsilon^{*} k_{f}+\varepsilon k_{d i s_{y y}}\right) \frac{\partial\langle T\rangle^{f}}{\partial r}\right|_{r=D_{1} / 2} \\ +\left.\left(1-\varepsilon^{*}\right) k_{s} \frac{\partial\langle T\rangle^{s}}{\partial r}\right|_{r=D_{1} / 2}\end{array}\right]=$ const.

$\left.\frac{\partial\langle T\rangle^{f}}{\partial r}\right|_{r=D_{2} / 2}=\left.\frac{\partial\langle T\rangle^{s}}{\partial r}\right|_{r=D_{2} / 2}=0$

\section{THEORITICAL APPROACHES FOR EXACT SOLU- TIONS}

For the sake of seeking possible exact solutions for convective heat transfer in an annulus filled with a porous media, Equations (11) and (12) are added to form,

$$
\begin{aligned}
& \rho_{f} c_{p_{f}} u_{D} \frac{\partial\langle T\rangle^{f}}{\partial x} \\
&=\frac{1}{r} \frac{\partial}{\partial r}\left(\begin{array}{r}
\left(\varepsilon^{*} k_{f}+\varepsilon k_{d i s_{y y}}\right) \\
r \frac{\partial\langle T\rangle^{f}}{\partial r} \\
\left.+\left(1-\varepsilon^{*}\right) k_{s} r \frac{\partial\langle T\rangle^{s}}{\partial r}\right)
\end{array}\right.
\end{aligned}
$$

where $u_{D}=\langle u\rangle$ is the Darcian velocity. Upon integrating the foregoing equation (15) across the cross-section of the annulus with the boundary conditions given by Equation (13) and (14), the energy balance readily gives,

$$
\rho_{f} c_{p_{f}} u_{D} \frac{d\langle T\rangle_{B}^{f}}{d x}=\frac{4 q_{w} D_{1}}{\left(D_{2}^{2}-D_{1}^{2}\right)}
$$

where $\langle T\rangle_{B}^{f}$ is the bulk temperature of the fluid phase. Hence,

$$
\frac{d\langle T\rangle_{B}^{f}}{d x}=\frac{\partial\langle T\rangle^{f}}{\partial x}=\frac{\partial\langle T\rangle^{s}}{\partial x}=\frac{4 q_{w} D_{1}}{\rho_{f} c_{p_{f}} u_{D}\left(D_{2}^{2}-D_{1}^{2}\right)}
$$


Note that the temperatures increase linearly downstream, although the velocity is unchanged. Equation (16) can be substituted into Equation (15) to give,

$$
\begin{aligned}
\frac{1}{r} \frac{\partial}{\partial r}\left(\left(\varepsilon^{*} k_{f}+\varepsilon k_{d i s_{y y}}\right) r \frac{\partial\langle T\rangle^{f}}{\partial r}+\left(1-\varepsilon^{*}\right) k_{s} r\right. & \left.\frac{\partial\langle T\rangle^{s}}{\partial r}\right) \\
= & 4 \frac{q_{w} D_{1}}{\left(D_{2}^{2}-D_{1}^{2}\right)}
\end{aligned}
$$

This may be integrated as,

$$
\begin{aligned}
\left(\varepsilon^{*} k_{f}+\varepsilon k_{d i i_{y y}}\right) \frac{\partial\langle T\rangle^{f}}{\partial r} & +\left(1-\varepsilon^{*}\right) k_{s} \frac{\partial\langle T\rangle^{s}}{\partial r} \\
& =2 \frac{q_{w} D_{1}}{\left(D_{2}^{2}-D_{1}^{2}\right)}\left(r-\frac{D_{2}^{2}}{4 r}\right)
\end{aligned}
$$

where the boundary conditions Equation (13) and (14) are exploited. The equation can further be integrated as,

$$
\begin{aligned}
\left(\varepsilon^{*} k_{f}+\varepsilon k_{d i s_{y y}}\right) & \left(\left(T_{w}-\Delta T\right)-\langle T\rangle^{f}\right) \\
+ & \left(1-\varepsilon^{*}\right) k_{s}\left(T_{w}-\langle T\rangle^{s}\right) \\
& =2 \frac{q_{w} D_{1}}{\left(D_{2}^{2}-D_{1}^{2}\right)}\left(\frac{D_{2}^{2}}{4} \ln \frac{2 r}{D_{1}}-\frac{r^{2}-D_{1}^{2} / 4}{2}\right)
\end{aligned}
$$

where, $\left.\langle T\rangle^{s}\right|_{r=D_{1} / 2}=T_{w}$ and $\left.\langle T\rangle^{f}\right|_{r=D_{1} / 2}=T_{w}-\Delta T$. The degree of thermal non-equilibrium, $\Delta T=\left.\left(\langle T\rangle^{s}-\langle T\rangle^{f}\right)\right|_{r=D_{1} / 2}$, must be prescribed. The foregoing relationship (17) between the solid and fluid temperatures is substituted into Equation (12) to obtain the following ordinary differential equation in terms of $\left(\langle T\rangle^{s}-T_{w}\right)$ as,

$$
\begin{gathered}
\left(1-\varepsilon^{*}\right) k_{s} \frac{1}{r} \frac{d}{d r}\left(r \frac{d\left(\langle T\rangle^{s}-T_{w}\right)}{\partial r}\right) \\
-\frac{k_{s t a g}+\varepsilon k_{d i s_{y y}}}{\varepsilon^{*} k_{f}+\varepsilon k_{d i s_{y y}}} h_{v}\left(\langle T\rangle^{s}-T_{w}\right) \\
=\frac{2 h_{v} q_{w} D_{1}}{\left(\varepsilon^{*} k_{f}+\varepsilon k_{d i s_{y y}}\right)\left(D_{2}^{2}-D_{1}^{2}\right)} \\
\left(\frac{D_{2}^{2}}{4} \ln \frac{2 r}{D_{1}}-\frac{r^{2}-D_{1}^{2} / 4}{2}\right)+h_{v} \Delta T
\end{gathered}
$$

Note that $\left(\langle T\rangle^{s}-T_{w}\right)$ is a function of $r$ alone, since $d T_{w} / d x=4 q_{w} D_{1} / \rho_{f} c_{p_{f}} u_{D}\left(D_{2}^{2}-D_{1}^{2}\right)$. This ordinary differential equation, after some manipulations, yields,

$$
\begin{aligned}
& \frac{\langle T\rangle^{s}-T_{w}}{D_{2} q_{w} /\left(k_{\text {stag }}+\varepsilon k_{d i s_{y y}}\right)} \\
& \quad=B_{1} I_{0}(\lambda r)+B_{2} K_{0}(\lambda r)+A r^{2}+C \ln \frac{2 r}{D_{1}}+E
\end{aligned}
$$

where,

$$
\begin{aligned}
& B_{1}=\frac{\left(A D_{2}+\frac{2 C}{D_{2}}\right) K_{0}\left(\lambda D_{1} / 2\right)-\left.\left(A \frac{D_{1}^{2}}{4}+E\right) \frac{d K_{0}(\lambda r)}{d r}\right|_{r=D_{2} / 2}}{\left.I_{0}\left(\lambda D_{1} / 2\right) \frac{d K_{0}(\lambda r)}{d r}\right|_{r=D_{2} / 2}-\left.K_{0}\left(\lambda D_{1} / 2\right) \frac{d I_{0}(\lambda r)}{d r}\right|_{r=D_{2} / 2}} \\
& B_{2}=\frac{\left(A D_{2}+\frac{2 C}{D_{2}}\right) I_{0}\left(\lambda D_{1} / 2\right)-\left.\left(A \frac{D_{1}^{2}}{4}+E\right) \frac{d I_{0}(\lambda r)}{d r}\right|_{r=D_{2} / 2}}{\left.K_{0}\left(\lambda D_{1} / 2\right) \frac{d I_{0}(\lambda r)}{d r}\right|_{r=D_{2} / 2}-\left.I_{0}\left(\lambda D_{1} / 2\right) \frac{d K_{0}(\lambda r)}{d r}\right|_{r=D_{2} / 2}} \\
& A=\frac{D_{1} / D_{2}}{D_{2}^{2}-D_{1}^{2}} \\
& C=-\frac{D_{1} D_{2} / 2}{D_{2}^{2}-D_{1}^{2}}
\end{aligned}
$$

$E=\frac{1}{\lambda^{2}}\left[\begin{array}{l}\frac{4 D_{1} / D_{2}}{D_{2}^{2}-D_{1}^{2}}-\frac{h_{v}}{\left(1-\varepsilon^{*}\right) k_{s}} \\ {\left[\begin{array}{c}\frac{\Delta T}{D_{2} q_{w} /\left(k_{s t a g}+\varepsilon k_{d i s_{y y}}\right)} \\ +\frac{D_{1}}{4 D_{2}} D_{1}^{2}\left(k_{s t a g}+\varepsilon k_{d i s_{y y}}\right) \\ \left(D_{2}^{2}-D_{1}^{2}\right)\left(\varepsilon^{*} k_{f}+\varepsilon k_{d i s_{y y}}\right)\end{array}\right]}\end{array}\right]$

$$
\lambda=\sqrt{\frac{\left(\frac{k_{s t a g}}{k_{f}}+\varepsilon \frac{k_{d i s_{y y}}}{k_{f}}\right)}{\left(\varepsilon^{*}+\varepsilon \frac{k_{d i s_{y y}}}{k_{f}}\right)\left(1-\varepsilon^{*}\right) \frac{k_{s}}{k_{f}}}\left(\frac{h_{v}}{k_{f}}\right)}
$$

Note that,

$$
\begin{aligned}
& \left.D_{2} \frac{d I_{0}(\lambda r)}{d r}\right|_{r=D_{2} / 2} \\
& =I_{1}\left(\lambda D_{2} / 2\right)\left(\frac{\lambda D_{2}}{2}-\frac{8}{\lambda D_{2}}\right)+\frac{\lambda D_{2}}{2} I_{3}\left(\lambda D_{2} / 2\right) \\
& +2\left(I_{0}\left(\lambda D_{2} / 2\right)+I_{2}\left(\lambda D_{2} / 2\right)\right)
\end{aligned}
$$




$$
\left.D_{2} \frac{d K_{0}(\lambda r)}{d r}\right|_{r=D_{2} / 2}=-\lambda D_{2} K_{1}\left(\lambda D_{2} / 2\right)
$$

where $I_{0,1,2,3}$ are the modified $I$ Bessel functions of the zeroth, first, second and third order, respectively. Correspondingly, $K_{0,1,2,3}$ are also the modified $K$ Bessel functions of the zeroth, first, second and third order, respectively.

The combination of Equation (19) and (17) readily gives the temperature of the fluid phase:

$$
\begin{gathered}
\frac{\langle T\rangle^{f}-T_{w}}{D_{2} q_{w} /\left(k_{\text {stag }}+\varepsilon k_{d i s_{y y}}\right)} \\
=\frac{\left(k_{\text {stag }}+\varepsilon k_{d i s_{y y}}\right) 2 D_{1}\left[\frac{1}{2}\left(r^{2}-\left(\frac{D_{1}}{2}\right)^{2}\right)-\left(\frac{D_{2}}{2}\right)^{2} \ln \frac{2 r}{\left.D_{1}\right]}\right.}{\left(\varepsilon^{*} k_{f}+\varepsilon k_{d i s_{y y}}\right) D_{2}\left(D_{2}^{2}-D_{1}^{2}\right)} \\
-\frac{\left(1-\varepsilon^{*}\right) k_{s}}{\varepsilon^{*} k_{f}+\varepsilon k_{d i s_{y y}}}\left(B_{1} I_{0}(\lambda r)+B_{2} K_{0}(\lambda r)+A r^{2}+C \ln \frac{2 r}{D_{1}}+E\right) \\
-\frac{\Delta T}{D_{2} q_{w} /\left(k_{\text {stag }}+\varepsilon k_{d i s_{y y}}\right)}
\end{gathered}
$$

As for the degree of thermal non-equilibrium, $\Delta T$, let us consider the two asymptotic conditions, namely, the local thermal equilibrium condition at the inner wall, i.e. $\Delta T=0$, and the local uniform heat flux condition at the inner wall, as given by,

$$
q_{w}=-\left.\frac{\varepsilon^{*} k_{f}+\varepsilon k_{d i s_{y}}}{\varepsilon} \frac{\partial\langle T\rangle^{f}}{\partial r}\right|_{r=D_{1} / 2}=-\left.\frac{1-\varepsilon^{*}}{1-\varepsilon} k_{s} \frac{\partial\langle T\rangle^{s}}{\partial r}\right|_{r=D_{1} / 2}
$$

which gives,

$$
\begin{gathered}
\frac{\Delta T}{D_{2} q_{w} /\left(k_{s t a g}+\varepsilon k_{d i s_{y y}}\right)} \\
=\frac{\frac{D_{1} / D_{2}}{\frac{1}{4}\left(1-\left(D_{1} / D_{2}\right)^{2}\right)}-E\left(\lambda D_{2}\right)^{2}}{h_{v} D_{2}^{2} /\left(1-\varepsilon^{*}\right) k_{s}} \\
-\frac{k_{s t a g}+\varepsilon k_{d i s_{y y}}}{\varepsilon^{*} k_{f}+\varepsilon k_{d i s_{y y}}} \frac{D_{1}^{3}}{4 D_{2}\left(D_{2}^{2}-D_{1}^{2}\right)}
\end{gathered}
$$

\section{POSSIBLE APPLICATIONS TO ALUMINUM FOAM AND AIR COMBINATION}

Due to a number of advantages such as high thermal conductivity, high strength, large specific surface area and low density etc., aluminum foams are quite ideal porous media for possible engineering applications, such as compact heat exchangers, heat sinks for power electronics, condenser towers and regenerators. Therefore, a large body of literature associated with aluminum foam is found. Some useful experimental correlations for the stagnant thermal conductivity, the volumetric heat transfer coefficient and the dispersion coefficient are proposed by Calmidi and Mahajan [23, 24]. These are listed as follows:

$\frac{k_{\text {stag }}}{k_{f}}=\varepsilon+0.19(1-\varepsilon)^{0.763} \sigma$

$N u_{v}=\frac{h_{v} d_{m}^{2}}{k_{f}}=8.72(1-\varepsilon)^{1 / 4}\left(\frac{1-e^{-(1-\varepsilon) / 0.04}}{\varepsilon}\right)^{1 / 2}\left(\frac{u_{D} d_{m}}{v}\right)^{1 / 2} \operatorname{Pr}^{0.37}$

$$
\frac{\varepsilon k_{d i s_{y y}}}{k_{f}}=0.06\left(\frac{\rho_{f} c_{p_{f}} u_{D} \sqrt{K}}{k_{f}}\right)
$$

where $u_{D}$ is the Darcian velocity, $\sigma$ is ratio of thermal conductivity of solid phase to that of fluid phase, and the permeability $K$ is given by the following empirical correlation [23]:

$K / d_{m}^{2}=0.00073(1-\varepsilon)^{-0.224}\left(\frac{1.18}{1-e^{-(1-\varepsilon) / 0.04}} \sqrt{\frac{1-\varepsilon}{3 \pi}}\right)^{-1.11}$

where $d_{m}$ is the pore diameter.

Both fluid and solid temperature profiles over the crosssection of the annulus for the case of aluminum foam and air combination, with $\sigma=8200, \varepsilon=0.95, \rho_{f} c_{p_{f}} u_{D} D_{2} / k_{f}=5000$, $d_{m} / D_{2}=0.1$ and $K / d_{m}^{2}=0.015$, are shown in Figs. (2a and b) for these two asymptotic cases, namely, the local thermal equilibrium inner wall case, i.e. $\Delta T=0$ and the local uniform heat flux inner wall case, respectively. The stagnant thermal conductivity, the volumetric heat transfer coefficient and the dispersion coefficient, obtained based on Equations (31) to (34) introduced previously, are given by $k_{\text {stag }} / k_{f}=160, \quad \varepsilon k_{d i s_{y y}} / k_{f}=3.67 \quad$ and $\quad \lambda D_{2}=42.1$, respectively.

In reality, the temperature difference between aluminum and air at the walls $\Delta T=\left(\langle T\rangle^{s}-\langle T\rangle^{f}\right)$, should be nonnegative. Therefore, the case of the local thermal equilibrium, as shown in Fig. (2a), is reasonable, since the base materials usually exhibit sufficiently high thermal conductivity. In a consolidated porous medium of metal foam, heat conducts through the solid phase. Therefore, the solid temperature is expected to be higher than the fluid temperature. However, the local thermal uniform heat flux condition at the inner wall, as illustrated in Fig. (2b), results 
in the negative $\Delta T=\left.\left(\langle T\rangle^{s}-\langle T\rangle^{f}\right)\right|_{r=D_{1} / 2}$, since, under such a condition, the fluid temperature gradient towards the wall becomes so high in order to generate the required uniform heat flux on the adjacent wall. This makes the fluid temperature exceed the solid temperature towards the wall. Obviously, this asymptotic condition may never be realized in practical applications. Nevertheless, both figures clearly indicate that the solid temperature in most region of the gap away from inner wall stays always substantially higher than the fluid temperature for these two cases discussed in this study, irrespective of the degree of thermal non-equilibrium $\Delta T$. Hence, the one energy equation model fails to be valid for these cases of the aluminum foam and air combination.

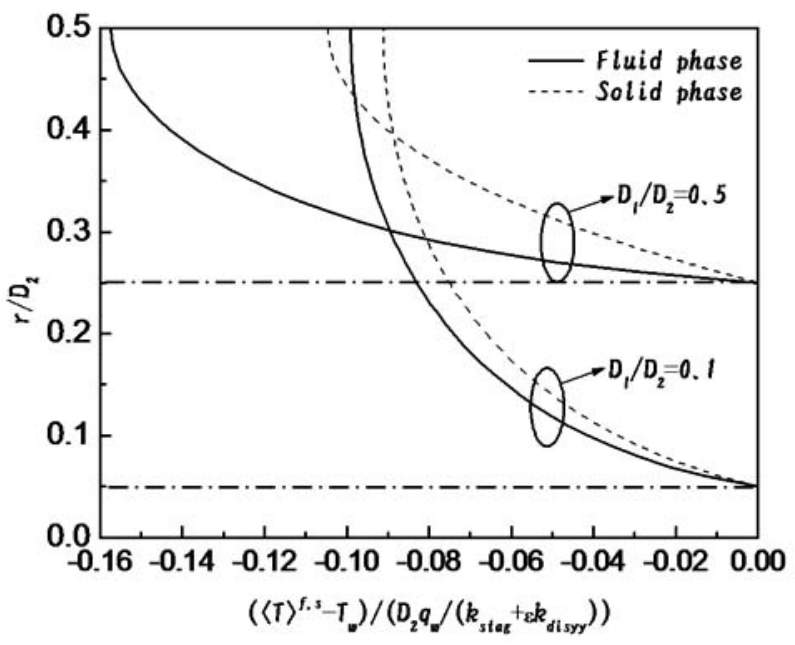

(a) Local thermal equilibrium at inner wall ( $\Delta T=0)$

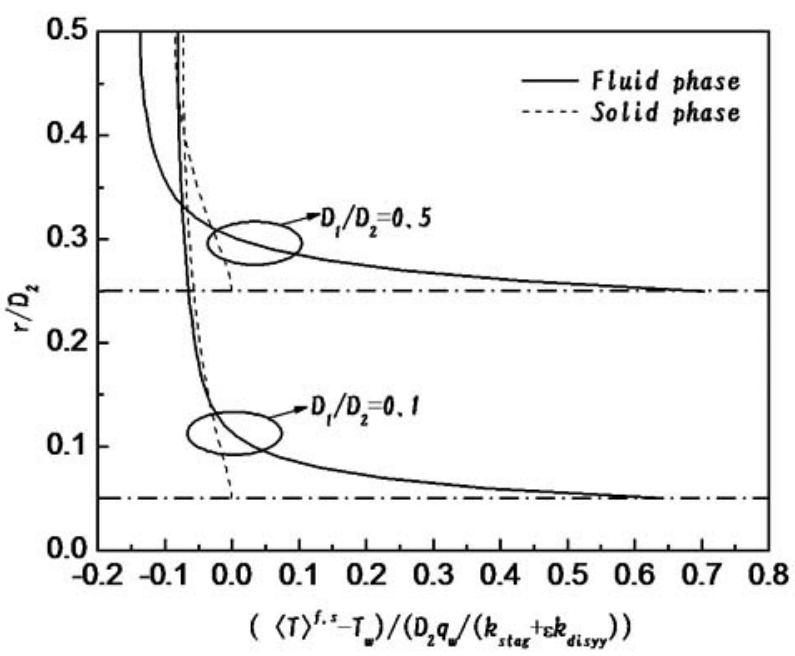

(b) Local uniform heat flux at inner wall

Fig. (2). Fluid and solid temperature profiles in an annulus filled with a metal foam. from,

The corresponding Nusselt number may be evaluated

$$
\begin{aligned}
N u_{D_{2}}= & \frac{q_{w} D_{2}}{\left(T_{w}-\langle T\rangle_{B}^{f}\right) k_{f}} \\
= & \frac{k_{\text {stag }}+\varepsilon k_{d i s_{y y}}}{k_{f}} \frac{1}{\frac{8}{D_{2}^{2}-D_{1}^{2}} \int_{D_{1} / 2}^{D_{2} / 2}\left(\frac{T_{w}-\langle T\rangle^{f}}{q_{w} D_{2} /\left(k_{\text {stag }}+\varepsilon k_{d i s_{y y}}\right)}\right) r d r}
\end{aligned}
$$

Note that the reference length for defining the Nusselt number can be any one of relevant dimensions, such as the inner radius, outer, inner and hydraulic radii. Equation (28) may be substituted into the foregoing equation to find the Nusselt number for the case of local thermal equilibrium at the wall ( $\Delta T=0)$, as shown in Fig. (3). The figure indicates that with the rise of Peclet number based on the pore diameter, Nusselt number substantially has a significant increase which may be persuasive for the possible engineering applications of aluminum foam and air combination.

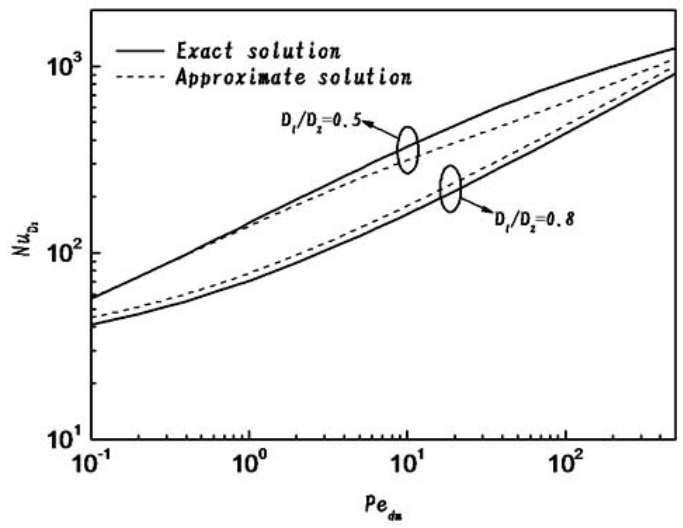

Fig. (3). Nusselt number for an annulus filled with a metal foam bounded by a local thermal equilibrium inner wall.

\section{APPROXIMATE ANALYSIS}

We shall seek a simple expression for Nusselt number for the case of local thermal equilibrium inner wall. An approximate treatment based on an integral method is presented here. We shall adopt Equation (18) which, for the case of local thermal equilibrium inner wall $(\Delta T=0)$, may be integrated as,

$$
\begin{aligned}
& -\eta \frac{d \theta}{d \eta}-\left(\frac{\lambda D_{1}}{2}\right)^{2} \int_{\eta}^{\alpha} \eta \theta d \eta \\
& =\frac{\left(\lambda D_{1} / 2\right)^{2}}{2 \alpha\left(\alpha^{2}-1\right)}\left[\begin{array}{r}
\alpha^{2}\left(\frac{\alpha^{2}}{2} \ln \alpha-\frac{1}{2} \eta^{2} \ln \eta-\frac{\alpha^{2}-\eta^{2}}{4}\right) \\
-\frac{1}{8}\left(\alpha^{4}-\eta^{4}\right)+\frac{1}{4}\left(\alpha^{2}-\eta^{2}\right)
\end{array}\right]
\end{aligned}
$$

Where,

$$
\theta(\eta)=\frac{\left(\langle T\rangle^{s}-T_{w}\right)\left(k_{\text {stag }}+\varepsilon k_{d i s_{y y}}\right)}{q_{w} D_{2}}
$$


$\eta=\frac{r}{\left(D_{1} / 2\right)}$

$\alpha=\frac{D_{2}}{D_{1}}$

Further integration leads to,

$-\theta(\alpha)-\left(\frac{\lambda D_{1}}{2}\right)^{2} \frac{\theta(\alpha)}{(\alpha-1)^{2}} \int_{1}^{\alpha}\left(\frac{1}{\eta} \int_{\eta}^{\alpha} \eta \theta d \eta\right) d \eta$

$=\frac{\left(\lambda D_{1} / 2\right)^{2}}{2 \alpha\left(\alpha^{2}-1\right)}\left[\begin{array}{c}\frac{\alpha^{4}}{2}(\ln \alpha)^{2}-\frac{\left(5 \alpha^{2}-2\right) \alpha^{2}}{8} \ln \alpha \\ +\frac{3\left(\alpha^{2}-1\right)\left(3 \alpha^{2}-1\right)}{32}\end{array}\right]$ as,

The solid phase temperature profile $\theta(\eta)$ may be assumed

$\theta(\eta)=\frac{\theta(\alpha)}{(\alpha-1)^{2}}\left(1-2 \alpha+2 \alpha \eta-\eta^{2}\right)$

Such that $\theta(1)=0$ and substitution of Equation (41) into (40) gives the unknown parameter:

$\theta(\alpha)$

$$
=-\frac{\frac{\left(\lambda D_{1} / 2\right)^{2}}{2 \alpha\left(\alpha^{2}-1\right)}\left[\frac{\alpha^{4}}{2}(\ln \alpha)^{2}-\frac{\left(5 \alpha^{2}-2\right) \alpha^{2}}{8} \ln \alpha\right.}{1+\frac{\left(\lambda D_{1} / 2\right)^{2}}{144(\alpha-1)^{2}}\left[\begin{array}{c}
12 \alpha^{2}\left(6-12 \alpha+5 \alpha^{2}\right) \ln \alpha+(\alpha-1) \\
\left.+\frac{32}{2}-1\right)
\end{array}\right]}
$$

From Equation (17), we find,

$$
\begin{aligned}
& \frac{T_{w}-\langle T\rangle^{f}}{D_{2} q_{w} /\left(k_{s t a g}+\varepsilon k_{d i s_{y y}}\right)} \\
& =\frac{\left(1-\varepsilon^{*}\right) k_{s} \theta(\eta)+\frac{k_{s t a g}+\varepsilon k_{d i s_{y y}}\left(\alpha^{2} \ln \eta-\frac{1}{2}\left(\eta^{2}-1\right)\right)}{2 \alpha\left(\alpha^{2}-1\right)}}{\varepsilon^{*} k_{f}+\varepsilon k_{d i s_{y y}}}
\end{aligned}
$$

Thus, the Nusselt number of our concern may be evaluated according to (35) and (43) as,
$N u_{D_{2}}=\frac{k_{s t a g}+\varepsilon k_{d i s_{y y}}}{k_{f}}$

$\left(\begin{array}{c}\left(1-\varepsilon^{*}\right) k_{s} \frac{\left(\alpha^{2}-1\right)(\alpha-1)^{2}}{2 \theta(\alpha)} \varepsilon_{f}+\varepsilon k_{d i s_{y y}} \\ {\left[\frac{(1-2 \alpha)\left(\alpha^{2}-1\right)}{2}+\frac{2 \alpha\left(\alpha^{3}-1\right)}{3}-\frac{\alpha^{4}-1}{4}\right]} \\ +\left(k_{s t a g}+\varepsilon k_{d i s_{y y}}\right) \frac{\left(\alpha^{4}\right.}{2} \ln \alpha+\frac{\left(\alpha^{2}-1\right)\left(1-3 \alpha^{2}\right)}{8}\end{array}\right]$

The approximate curve for the Nusselt number based on Equation (44) is presented in Fig. (3), which shows reasonably good agreement with the exact curve.

\section{CONCLUSIONS}

Using a local thermal non-equilibrium model, thermally fully developed flow in an annulus filled with a porous medium was investigated in this study, where inner and outer walls are subject to constant heat flux and adiabatic respectively. Exact solutions for fluid and solid phases were obtained for these two asymptotic cases, namely, the local thermal equilibrium case and the local uniform heat flux case at the inner wall, respectively. An aluminum foam and air combination was chosen as an illustrative example in views of its possible engineering application potential. The temperature profiles achieved for the aluminum and air phases are quite different, which means the utilization of local thermal equilibrium assumption may lead to large discrepancies for the cases. An approximate solution was presented to evaluate the Nusselt number for the case of local thermal equilibrium at the inner wall, which is found in accord with the corresponding exact solution.

\section{ACKNOWLEDGEMENTS}

This work has been partially supported by the National Natural Science Foundation of China (No. 51036003). 


\section{ABBREVIATIONS}

$$
\begin{aligned}
& A \quad=\text { Surface area }\left(\mathrm{m}^{2}\right) \\
& A_{\text {int }}=\text { Interface between the fluid and solid }\left(\mathrm{m}^{2}\right) \\
& c \quad=\text { Specific heat }(\mathrm{J} / \mathrm{kgK}) \\
& c_{p}=\text { Specific heat at constant pressure }(\mathrm{J} / \mathrm{kgK}) \\
& D_{l} \quad=\text { Tube inner diameter }(\mathrm{m}) \\
& D_{2} \quad=\text { Tube outer diameter }(\mathrm{m}) \\
& d_{m}=\text { Mean pore diameter }(\mathrm{m}) \\
& h_{v}=\text { Volumetric heat transfer coefficient }\left(\mathrm{W} / \mathrm{m}^{3} \mathrm{~K}\right) \\
& k=\text { Thermal conductivity }(\mathrm{W} / \mathrm{mK}) \\
& K=\text { Permeability }\left(\mathrm{m}^{2}\right) \\
& n_{j}=\text { Unit vector pointing outward from the fluid side } \\
& \text { to solid side (-) } \\
& \operatorname{Pr} \quad=\text { Prandtl number (-) } \\
& q \quad=\text { Heat flux }\left(\mathrm{W} / \mathrm{m}^{2}\right) \\
& r \quad=\text { Radial coordinate } \\
& T \quad=\text { Temperature }(\mathrm{K}) \\
& u_{D}=\text { Darcian velocity (Uniform inlet velocity) }(\mathrm{m} / \mathrm{s}) \\
& u_{i}=\text { Velocity vector }(\mathrm{m} / \mathrm{s}) \\
& V=\text { Representative elementary volume }\left(\mathrm{m}^{3}\right) \\
& x_{i}=\text { Cartesian coordinates }(\mathrm{m}) \\
& x, y, z=\text { Cartesian coordinates }(\mathrm{m}) \\
& \varepsilon \quad=\text { Porosity (-) } \\
& \varepsilon^{*}=\text { Effective porosity (-) } \\
& v=\text { Kinematic viscosity }\left(\mathrm{m}^{2} / \mathrm{s}\right) \\
& \rho \quad=\text { Density }\left(\mathrm{kg} / \mathrm{m}^{2}\right) \\
& \alpha \quad=\quad \text { Ratio of tube outer diameter } D_{2} \text { to tube inner }
\end{aligned}
$$

\section{Special Symbols}

$\widetilde{\phi}=$ Deviation from intrinsic average

$\langle\phi\rangle=$ Darician average

$\langle\phi\rangle^{f, s}=$ Intrinsic average

\section{Subscripts and Superscripts}

$$
\begin{array}{ll}
\text { dis } & =\text { Dispersion } \\
f & =\text { Fluid } \\
s & =\text { Solid } \\
\text { stag } & =\text { Stagnation } \\
w & =\text { Inner wall }
\end{array}
$$

\section{REFERENCES}

[1] C. Yang, W. Liu, and A. Nakayama, "Forced convective heat transfer enhancement in a tube with its core partially filled with a porous medium" Open Transport Phenomena J., vol. 1, pp. 1-6, 2009

[2] T. M. Jeng, S. C. Tzeng, and Y. H. Hung, "An analytical study of local thermal equilibrium in porous heat sinks using fin theory", Int. J. Heat Mass Transfer, vol. 49, pp. 1907-1914, 2006.

[3] P. Vadasz, "Explicit conditions for local thermal equilibrium in porous media heat conduction", Transport Porous Media, vol. 59, pp. 341-355, 2005.

[4] R. G. Carbonnel, and S. Whitaker, "Heat and mass transfer in porous media", In: Bear, J., Corapcioglu, M.Y. (Eds.), Fundamentals of Transport Phenomena in Porous Media, Martinus Nijhoff, Dordrecht (Boston), pp. 121-198, 1984.

[5] K. Vafai, and M. Sozen, "Analysis of energy and momentum transport for fluid flow through a porous bed", Heat Transfer, vol. 112, pp. 690-699, 1990.

[6] M. Quintard, and S. Whitaker, "One and two equation models for transient diffusion processes in two-phase systems", Adv. Heat Transfer, vol. 23, pp. 369-465, 1993.

[7] M. Quintard, and S. Whitaker, "Local thermal equilibrium for transient heat conduction: theory and comparison with numerical experiments", Int. J. Heat Mass Transfer, vol. 38, pp. 2779-2796, 1995.

[8] M. Quintard, "Modelling local non- equilibrium heat transfer in porous media", Proc. 11th Int. Heat Transfer Conf., vol. 1, pp. 279285, 1998.

[9] A. Amiri, and K. Vafai, "Analysis of dispersion effects and nonthermal equilibrium, non-Darcian, variable porosity incompressible flow through porous media", Int. J. Heat Mass Transfer, vol. 37, pp. 939-954, 1994

[10] P. X. Jiang, Z. P. Ren, B. X. Wang, and Z. Wang, "Forced convective heat transfer in a plate channel filled with solid particles", J. Thermal Sci., vol. 5, pp. 43-53, 1996.

[11] P. X. Jiang, and Z. P. Ren, "Numerical investigation of forced convection heat transfer in porous media using a thermal nonequilibrium model", Int. J. Heat Fluid Flow, vol. 22, pp. 102-110, 2001

[12] G. P. Peterson, and C. S. Chang, "Two-phase heat dissipation utilizing porous-channels of high-conductivity materials", J. Heat Transfer, vol. 120, pp. 243-252, 1998.

[13] M. Spiga, and G. L. Morini, "Transient response of non-thermal equilibrium packed beds", Int. J. Eng. Sci., vol. 37, pp. 179-188, 1999.

[14] B. Alazmi, and K. Vafai, "Constant wall heat flux boundary conditions in porous media under local thermal non-equilibrium conditions", Int. J. Heat Mass Transfer, vol. 45, pp. 3071-3087, 2002.

[15] C. T. Hsu, "Heat conduction in porous media", in: K. Vafai (Ed.), Handbook of Porous Media, Marcel Dekker, New York, pp. 170 200, 2000.

[16] C. T. Hsu, P. Cheng, and K. W. Wong, "A lumped parameter model for stagnant thermal conductivity of spatially periodic porous media", ASME Trans. J. Heat Transfer, vol. 117, pp. 264$269,1995$.

[17] A. Nakayama, F. Kuwahara, M. Sugiyama, and G. L. Xu, "A Twoenergy equation model for conduction and convection in porous media", Int. J. Heat Mass Transfer, vol. 44, no. 22, pp. 4375-4379, 2001.

[18] P. Cheng, "Heat transfer in geothermal systems", Adv. Heat Transfer, vol. 14, pp. 1-105, 1978.

[19] A. Nakayama, PC-aided numerical heat transfer and convective flow, CRC Press, Boca, Raton, 1995, pp. 49-50, 103-115.

[20] C. Yang, and A. Nakayama, "A synthesis of tortuosity and dispersion in effective thermal conductivity of porous media", Int. J. Heat Mass Transfer, vol. 53, pp. 3222-3230, 2010.

[21] A. Nakayama, F. Kuwahara, and Y. Kodama, "An equation for thermal dispersion flux transport and its mathematical modelling for heat and fluid flow in a porous medium", J. Fluid Mechanics, vol. 563, pp. 81-96, 2006.

[22] N. Dukhan, R. Picon-Feliciano, and A. R. Alvarez-Hernandez, "Heat transfer analysis in metal foams with low-conductivity fluids", J. Heat Transfer, vol. 128, pp. 784-792, 2006. 
[23] V. V. Calmidi, and R. L. Mahajan, "The effective thermal conductivity of high porosity fibrous metal foams", ASME Trans. $J$. Heat Transfer, vol. 121, pp. 466-471, 1999.
[24] V. V. Calmidi, and R. L. Mahajan, "Forced convection in high porosity metal foams", ASME Trans. J. Heat Transfer, vol. 122, pp. $557-565,2000$.

Received: April 07, 2011

Revised: May 18, 2011

Accepted: May 19, 2011

(C) Yang et al.; Licensee Bentham Open.

This is an open access article licensed under the terms of the Creative Commons Attribution Non-Commercial License (http://creativecommons.org/ licenses/by-nc/3.0/), which permits unrestricted, non-commercial use, distribution and reproduction in any medium, provided the work is properly cited. 